\title{
Alternativity and reciprocity in the Cayley-Dickson algebra
}

\author{
S. Kuwata, H. Fujii, and A. Nakashima \\ Faculty of Information Sciences, Hiroshima City University, \\ Asaminami-ku, Hiroshima 731-3194, Japan
}

\begin{abstract}
We calculate the eigenvalue $\rho$ of the multiplication mapping $R$ on the Cayley-Dickson algebra $\mathbb{A}_{n}$. If the element in $\mathbb{A}_{n}$ is composed of a pair of alternative elements in $\mathbb{A}_{n-1}$, half the eigenvectors of $R$ in $\mathbb{A}_{n}$ are still eigenvectors in the subspace which is isomorphic to $\mathbb{A}_{n-1}$. The invariant under the reciprocal transformation $\mathbb{A}_{n} \times \mathbb{A}_{n} \ni(x, y) \mapsto(-y, x)$ plays a fundamental role in simplifying the functional form of $\rho$. If some physical field can be identified with the eigenspace of $R$, with an injective map from the field to a scalar quantity (such as a mass) $m$, then there is a one-to-one map $\pi: m \mapsto \rho$. As an example, the electro-weak gauge field can be regarded as the eigenspace of $R$, where $\pi$ implies that the W-boson mass is less than the Z-boson mass, as in the standard model.
\end{abstract}

\section{Introduction}

The Cayley-Dickson algebra $\mathbb{A}_{n}$ over a field $F$ is the algebra structure $F^{2^{n}}$ given inductively by a pair of $\mathbb{A}_{n-1}$ through the Cayley-Dickson process [1]. If the base field $F$ is taken as the real number $\mathbb{R}, \mathbb{A}_{1}, \mathbb{A}_{2}$, and $\mathbb{A}_{3}$ correspond to the complex number $\mathbb{C}$, the quaternion $\mathbb{H}$, and the octonion $\mathbb{O}$, respectively. For $n<4, \mathbb{A}_{n}$ satisfies the alternative law: $x^{2} y=x(x y)$ and $x y^{2}=(x y) y$, so that $\mathbb{A}_{n}$ is a composition algebra, $\|x y\|=\|x\|\|y\|$ for all $x, y$ in $\mathbb{A}_{n}(n<4)$, that is, a normed division algebra. Thus, the multiplication map $R_{x}: \mathbb{A}_{n} \rightarrow \mathbb{A}_{n}$ by $R_{x}: y \mapsto y x$ (with $\|x\|=1$ ) belongs to $S O\left(2^{n}\right)$ for $n<4$.

The reciprocal transformation is given by $\mathbb{A}_{n} \times \mathbb{A}_{n} \ni(x, y) \mapsto(-y, x)$. Originally, the reciprocal transformation was given by the rotation of the phase space as $(q, p) \mapsto(-p, q)$, where $q, p$ represent position and momentum, respectively. As emphasized by Born 2, the law of nature is invariant under the reciprocal transformation. For example, the bosonic hamiltonian for a harmonic oscillator $\frac{1}{2}\left(q^{2}+p^{2}\right)$, the fermionic one $\frac{i}{2}(q p-p q)$, and the corresponding (anti)commutation relations $([q, p]=i,[q, q]=[p, p]=0)$ and $(\{q, p\}=0,\{q, q\}=\{p, p\}=1)$ are all invariant under $(q, p) \mapsto(-p, q)$. Thus, 
the invariant under the reciprocal transformation plays a fundamental role in restricting the theory.

For a physical application, $\mathbb{A}_{n}$ has been utilized by many authors [3, 4, 5, 6, 17, 8, 9, 10, 11. However, most of the studies are based on the division algebra (except in Ref. 12, where it is discussed that the gravitational force may be embedded in the enlarged space of $\left.\mathbb{A}_{4}\right)$. One of the reasons of adopting $\mathbb{A}_{n}$ for $n<4$ is that it is closely related to the (simple) Lie group. The simple Lie groups are categorized into two types: classical and exceptional ones. The classical ones, denoted by $S O(n), S U(n)$, and $S p(n)$, represent the isometric transformation in a $n$-dimensional vector space over $\mathbb{R}, \mathbb{C}$, and $\mathbb{H}$, respectively. Note that the isometry over $\mathbb{O}$ is not a Lie group, due to the lack of associativity; the multiplication over $\mathbb{O}$ does not form a group. However, $\mathbb{O}$ is still a composition algebra due to alternativity, so that $\mathbb{O}$ is related to the simple Lie group of an exceptional type as 13 .

$$
\begin{aligned}
& G_{2} \cong \operatorname{Aut}(\mathbb{O}), F_{4} \cong \operatorname{Isom}\left(\mathbb{O P}^{2}\right), \\
& E_{6} \cong \operatorname{Isom}\left((\mathbb{C} \otimes \mathbb{O}) \mathbb{P}^{2}\right), E_{7} \cong \operatorname{Isom}\left((\mathbb{H} \otimes \mathbb{O}) \mathbb{P}^{2}\right), E_{8} \cong \operatorname{Isom}\left((\mathbb{O} \otimes \mathbb{O}) \mathbb{P}^{2}\right),
\end{aligned}
$$

where Aut, Isom and $\mathbb{K} \mathbb{P}^{2}$ represent the automorphism, isometry and the projective plane over $\mathbb{K}$, respectively. For $n>3, \mathbb{A}_{n}$ is not directly related to the simple Lie algebra. This conversely implies that $\mathbb{A}_{n}$ (for $n>3$ ) is a natural candidate of an algebra for describing the breaking of the isometry of the simple Lie group.

The aim of this paper is first to obtain the isometry (or rotational symmetry) breaking of the multiplication map $R_{x}$, and then to apply the result to some rotational symmetry breaking system. If the field (or state) of the isometry breaking system can be identified with the eigenspace of $R_{x}$, with an injective map from the field and some scalar quantity $q$ (called an "order parameter"), then it is found that there is a one-to-one map $\pi$ from $q$ to the eigenvalue of $R_{x}$. As an example, we will show that the electro-weak gauge field can be regarded as the eigenspace of $R_{x}$. In this case, the one-to-one map $\pi$ between $q$ (here, the gauge boson mass) and the eigenvalue of $R_{x}$ implies that (under some reasonable condition) the W-boson mass is less than the Z-boson mass, as in the standard model.

In Sec. 2, we first review the basic properties of the Cayley-Dickson algebra, and then calculate the eigenvalue of $R_{(x, y)}$, to find that half the eigenvectors of $R_{(x, y)}$ in $\mathbb{A}_{n+1}$ (for $n<4$ ) are still eigenvectors in the subspace which is isomorphic to $\mathbb{A}_{n}$. This is due to the alternativity of $x$ and $y$ in $\mathbb{A}_{n}$. To guarantee this property for all $n$, we require that the element in $\mathbb{A}_{n+1}$ should be composed of a pair of alternative elements in $\mathbb{A}_{n}$. In Sec. 3 , we show how the invariant under the reciprocal transformation simplifies the functional form of the eigenvalue. In Sec. 4, we find that the eigenvalue of $R_{x}$ can be interpreted as the order parameter in some isometry breaking field, as long as the field can be identified with the eigenspace of $R_{x}$. In Sec. 5, we give summary. 


\section{Cayley-Dickson algebra}

\section{$2.1 \quad$ Basic property}

We review the basic properties of the Cayley-Dickson algebra $\mathbb{A}_{n}$ over the real numbers $\mathbb{R}$. For $x=\left(x_{1}, x_{2}\right)$ and $y=\left(y_{1}, y_{2}\right)$ in $\mathbb{R}^{2^{n}}=\mathbb{R}^{2^{n-1}} \times \mathbb{R}^{2^{n-1}}$, the multiplication is inductively given by [1, 14]

$$
x y=\left(x_{1} y_{1}-\bar{y}_{2} x_{2}, y_{2} x_{1}+x_{2} \bar{y}_{1}\right), \quad \text { with } \bar{x}=\left(\bar{x}_{1},-x_{2}\right),
$$

so that $\overline{x y}=\bar{y} \bar{x}$ for all $x, y$ in $\mathbb{A}_{n}$. The Euclidean norm and the inner product in $\mathbb{R}^{2^{n}}$ are given by

$$
\begin{aligned}
\|x\|^{2} & =x \bar{x}=\bar{x} x, \\
\langle x, y\rangle & =\frac{1}{2}(x \bar{y}+y \bar{x})=\Re(x \bar{y}),
\end{aligned}
$$

respectively, where $\Re(x)$ represents the real part of $x$, that is, $\Re(x)=\frac{1}{2}(x+\bar{x})$. For $n=0,1,2$, and $3, \mathbb{A}_{n}$ represents $\mathbb{R}, \mathbb{C}, \mathbb{H}$, and $\mathbb{O}$, respectively.

Notation 1 Denote the commutator by $[x, y]=x y-y x$, and the associator by $[x, y, z]=(x y) z-x(y z)$.

The basic properties of $\mathbb{A}_{n}$ are summarized in Table 1. Due to the flexibility of $\mathbb{A}_{n}$, we obtain for all $x, y, z$ in $\mathbb{A}_{n}$ the following identity [14]

$$
\langle x, y z\rangle=\langle x \bar{z}, y\rangle=\langle\bar{y} x, z\rangle .
$$

Moreover, the flexibility leads to 15

$$
\bar{x}(x y)=(y \bar{x}) x,
$$

which is less popular, compared to the flexible law. However, Eq. (3) is useful in obtaining Eq. (6).

Notation 2 For $x$ in $\mathbb{A}_{n}$, the right and left multiplications are denoted by $R_{x}$ and $L_{x}$, respectively, that is, $R_{x}, L_{x}: \mathbb{A}_{n} \rightarrow \mathbb{A}_{n}$ by

$$
y R_{x}=y x, \quad y L_{x}=x y .
$$

Table 1: Basic properties of $\mathbb{A}_{n}$.

\begin{tabular}{ccc}
\hline$n$ & Property & Identity \\
\hline 0 & Self-conjugate & $x=\bar{x}$ \\
0,1 & Commutative & {$[x, y]=0$} \\
$0,1,2$ & Associative & {$[x, y, z]=0$} \\
$0,1,2,3$ & Alternative & {$[x, x, y]=0$} \\
All & Flexible & {$[x, y, x]=0$} \\
\hline
\end{tabular}


Some may feel somewhat uneasy about the notation like $y R_{x}$, rather than a usual notation like $R_{x}(y)$. If we regard $y \in \mathbb{A}_{n}=\mathbb{R}^{2^{n}}$ as a row (not column) vector in $\mathbb{R}^{2^{n}}$, and $R_{x}$ as the corresponding $2^{n} \times 2^{n}$ matrix, $y R_{x}=y x$ corresponds to $\langle y| R_{x}=\langle y x|$ in the Dirac notation. From the recursion formula of Eq. (11), $R_{x}$ and $L_{x}$ are decomposed into

$$
R_{x}=\left(\begin{array}{cc}
R_{x_{1}} & L_{x_{2}} \\
-L_{\bar{x}_{2}} & R_{\bar{x}_{1}}
\end{array}\right), \quad L_{x}=\left(\begin{array}{cc}
L_{x_{1}} & \eta L_{x_{2}} \\
-\eta R_{x_{2}} & R_{x_{1}}
\end{array}\right)
$$

where $\eta: \mathbb{A}_{n} \rightarrow \mathbb{A}_{n}$ by $x \eta=\bar{x}$, so that the corresponding representation matrix is given by $\operatorname{diag}(1,-1,-1, \ldots,-1)$. From Eq. (4), it is found that

$$
{ }^{t} R_{x}=R_{\bar{x}}, \quad{ }^{t} L_{x}=L_{\bar{x}}
$$

where the superscript $t$ represents the transposition.

Now we examine whether $R_{x}$ (with $\|x\|=1$ ) belongs to $S O\left(2^{n}\right)$ or not. In this case, it is sufficient to analyze the algebra structure of $R_{x} R_{\bar{x}}$ instead of $R_{x}$ itself. This is because we have $\langle y, y\rangle \rightarrow\left\langle y R_{x}, y R_{x}\right\rangle=\left\langle y,\left(y R_{x}\right) R_{\bar{x}}\right\rangle=$ $\left\langle y, y\left(R_{x} R_{\bar{x}}\right)\right\rangle$ from Eq. (2). Since $R_{x} R_{\bar{x}}\left(=R_{x}{ }^{t} R_{x}\right)$ is positive semidefinite in $\mathbb{R}^{2^{n}}$, its eigenvalue turns out to be (non-negative) real, so that the vector space $\mathbb{R}^{2^{n}}$ can be decomposed into the eigenspace of $R_{x} R_{\bar{x}}$. If $y$ is the eigenvector of $R_{x} R_{\bar{x}}$ (with its eigenvalue $\nu$ ), then we obtain $\|y\|^{2} \rightarrow \nu\|y\|^{2}$, so that the violation of $R_{x} \in S O\left(2^{n}\right)$ can be checked through the relation of $\nu \neq 1$. In what follows, we will obtain the eigenvalue (together with the corresponding eigenvector) of $R_{x} R_{\bar{x}}$.

Notation 3 For $x$ in $\mathbb{A}_{n}, N_{x}=R_{x} R_{\bar{x}}$ and $\bar{N}_{x}=L_{x} L_{\bar{x}}$.

We have the following identity for all $x$ in $\mathbb{A}_{n}$ :

$$
N_{x}=N_{\bar{x}}=\bar{N}_{x}=\bar{N}_{\bar{x}}
$$

where $N_{\bar{x}}=\bar{N}_{x}$ is derived from Eq. (3). Substituting Eq. (4) into $N_{x}$ and using Eq. (6), we obtain 16.

$$
N_{x}=\left(\begin{array}{cc}
A_{x_{1}, x_{2}} & -B_{x_{1}, x_{2}} \\
B_{x_{1}, x_{2}} & A_{x_{1}, x_{2}}
\end{array}\right),
$$

where $A_{x_{1}, x_{2}}=N_{x_{1}}+N_{x_{2}}$ and $B_{x_{1}, x_{2}}=\left[R_{x_{1}}, L_{x_{2}}\right]$. Using the determinant identity $\left|\begin{array}{cc}A & -B \\ B & A\end{array}\right|=|A+i B||A-i B|=\left.|A+i B|\right|^{t} A-i^{t} B \mid$, and noticing that $A_{x_{1}, x_{2}}$ is symmetric and that $B_{x_{1}, x_{2}}$ is antisymmetric, we find that

$$
\left|N_{x}-\nu I\right|=\left|C_{x_{1}, x_{2}}-\nu I\right|^{2}
$$

where $C_{x_{1}, x_{2}}=A_{x_{1}, x_{2}}+i B_{x_{1}, x_{2}}$, so that the eigenvalue of $N_{x}$ is equal to that of $C_{x_{1}, x_{2}}$ [while the corresponding eigenvector $r$ of $N_{x}$, which is doubly degenerate, is given by $r=\left(d_{1}, d_{2}\right)$ and $\left(d_{2},-d_{1}\right)$, where $d_{1}$ and $d_{2}$ represent the real and imaginary parts of the eigenvector of $C_{x_{1}, x_{2}}$, respectively].

Thus, in the following, we concentrate on the calculation of the eigenvalue of $C_{x_{1}, x_{2}}$. 
Notation 4 For $x, y$ in $\mathbb{A}_{n}$, we set $C_{x, y}^{\prime}=C_{x, y}-\left(\|x\|^{2}+\|y\|^{2}\right) I$, where $\|x\|^{2}+$ $\|y\|^{2}$ represents the diagonal elements of $C_{x, y}$, which is obtained inductively. We also denote by $S_{n}$ the set of eigenvalues of $C_{x, y}^{\prime}$. For later convenience, if the element $s_{i} \in S_{n}$ has the same functional form as $s_{i}^{\prime} \in S_{n^{\prime}}\left(n^{\prime} \neq n\right)$, we regard them as the same element.

For $x, y$ in $\mathbb{A}_{n}$ with $n<4, C_{x, y}^{\prime}$ turns out to be $i B_{x, y}$. For $n<3, B_{x, y}$ is vanishing due to the associativity of $\mathbb{A}_{n}$, so that all the elements of $S_{n}$ is vanishing. For $n=3, S_{n}$ is calculated in a somewhat brute-force way [16. To summarize, we have

$$
\begin{aligned}
& S_{0}=\{0\}, \\
& S_{1}=\{0,0\}, \\
& S_{2}=\{0,0,0,0\}, \\
& S_{3}=\{0,0,0,0, \pm 2|\mathbf{x} \times \mathbf{y}|, \pm 2|\mathbf{x} \times \mathbf{y}|\},
\end{aligned}
$$

where $|\mathbf{x} \times \mathbf{y}|^{2}=\|\mathbf{x}\|^{2}\|\mathbf{y}\|^{2}-\langle\mathbf{x}, \mathbf{y}\rangle^{2}$, with the bold face letter $\mathbf{x}$ representing the imaginary part of $x$, that is, $\mathbf{x}=x-\Re(x)$. For $x \in \mathbb{A}_{n}$ (with $n<4$ ), it is found from Eq. (8) that all the eigenvalues of $N_{x}$ are given by $\|x\|^{2}$, due to the vanishing of all the elements of $S_{0}, S_{1}$ and $S_{2}$. Thus, we obtain $\|x y\|^{2}=$ $\left\langle x, x N_{y}\right\rangle=\langle x, x\rangle\|y\|^{2}=\|x\|^{2}\|y\|^{2}$ for all $x, y$ in $\mathbb{A}_{n}$ (with $n<4$ ). This indicates that $\mathbb{A}_{n}$ (for $n<4$ ) is the normed division algebra, as is well known.

It should be noted that the sets $S_{n}$ (for $n<4$ ) satisfy the following inclusion relation:

$$
S_{0} \subset S_{1} \subset S_{2} \subset S_{3}
$$

However, $S_{n-1} \subset S_{n}$ does not, in general hold for $n \geq 4$ in general; there is a counterexample. The relation of $S_{n-1} \subset S_{n}$ implies that half of the eigenvectors of $N_{(x, y)}$ in $\mathbb{A}_{n+1}$ are still eigenvectors in the subspace $V^{\prime}=\{(x, y)=$ $\left.\left(x_{1}, x_{2}, y_{1}, y_{2}\right) \in \mathbb{A}_{n}^{2}=\mathbb{A}_{n-1}^{4} \mid x_{2}=y_{2}=0\right\}$, which is isomorphic to $\mathbb{A}_{n}$. To be more strict, let $(p, q)=\left(p_{1}, p_{2} ; q_{1}, q_{2}\right)$ in $\mathbb{A}_{n}^{2}=\mathbb{A}_{n-1}^{4}$ be the eigenvector of $N_{(x, y)}$ with its eigenvalue $\nu_{x, y}$, that is, $(p, q) N_{(x, y)}=\nu_{x, y}(p, q)$. In the subspace $V^{\prime}$, the eigenequation turns out to be $\left(p_{1}, q_{1}\right) N_{\left(x_{1}, y_{1}\right)}=\nu_{x, y}^{\prime}\left(p_{1}, q_{1}\right)$, where $\nu_{x, y}^{\prime}=\lim _{x_{2}, y_{2} \rightarrow 0} \nu_{x, y}$. Suppose that $\left[\nu_{x, y}-\left(\|x\|^{2}+\|y\|^{2}\right)\right] \in S_{n}$ belongs also to $S_{n-1}$. Then, we find that $\lim _{x_{2}, y_{2} \rightarrow 0} \nu_{x, y}$ is the same functional form of $\nu_{x, y}$ where $x$ and $y$ are replaced by $x_{1}$ and $y_{1}$, respectively, because the element in $S_{n}$ having the same functional form as the element in $S_{n-1}$ is regarded as the same. Thus, we obtain $\nu_{x, y}^{\prime}=\nu_{x_{1}, y_{1}}$, which indicates that the eigenvector of $N_{(x, y)}$ in $\mathbb{A}_{n+1}$ is still the eigenvector of $N_{\left(x_{1}, y_{1}\right)}$ in the subspace $V^{\prime}\left(\cong \mathbb{A}_{n}\right)$, as long as $S_{n-1} \subset S_{n}$.

In the following subsection, we deal with the case where $S_{n-1} \subset S_{n}$ is satisfied for all $n$. This guarantees that half of the eigenvectors of $N_{(x, y)}$ in $\mathbb{A}_{n+1}$ remain the eigenvectors in the subspace which is isomorphic to $\mathbb{A}_{n}$. 


\section{$2.2 \quad$ Alternative entry}

Recall that for $n<4$, where $S_{n-1} \subset S_{n}$ is satisfied, $\mathbb{A}_{n}$ is alternative; recall also that for $n \geq 4$, where $S_{n-1} \subset S_{n}$ does not hold in general, $\mathbb{A}_{n}$ is not alternative. This implies that if the elements $x, y$ are "alternative" in $\mathbb{A}_{n}$, the inclusion relation $S_{n-1} \subset S_{n}$ is satisfied. Fortunately, this is indeed true, which will be proven in the following.

Definition 5 The element $x$ in $\mathbb{A}_{n}$ is alternative, if $[a, a, x]=0$ for all $x$ in $\mathbb{A}_{n}$.

Lemma 6 For $x, y$ in $\mathbb{A}_{n}($ for $n \geq 3)$,

$$
\mathbf{x} \| \mathbf{y} \Leftrightarrow B_{x, y}=0,
$$

where $\mathbf{x}=x-\Re(x), \mathbf{y}=y-\Re(y)$, and $\mathbf{x} \| \mathbf{y}$ represents $|\mathbf{x} \times \mathbf{y}|=0$.

Proof. $\Rightarrow)$ Since $B_{x, y}$ is independent of $\Re(x)$ and $\Re(y)$, there is no loss of generality that we can set $y=k x$ (or $x=k y$ ), where $k \in \mathbb{R}$. Due to the flexibility of $\mathbb{A}_{n}$, we find that $B_{x, x}=0$ for all $x$ in $\mathbb{A}_{n}$. Thus, we obtain $B_{x, y}=k B_{x, x}\left(\right.$ or $\left.k B_{y, y}\right)=0$.

$\Leftarrow)$ Notice that $B_{x, y}=0 \Leftrightarrow \forall z \in \mathbb{A}_{n},[y, z, x]=0$. Then, we show the contraposition such that if $\mathbf{x}+\mathbf{y}$, then $\exists z \in \mathbb{A}_{n},[y, z, x] \neq 0$. From $\mathbf{x} * \mathbf{y}$, it follows that both $\mathbf{x}$ and $\mathbf{y}$ are nonzero, so that there is no loss of generality that we can choose $\mathbf{x}=e_{1}$ and $\mathbf{y}=c_{1} e_{1}+c_{2} e_{2}$, where $c_{1}, c_{2} \in \mathbb{R}$ with $c_{2} \neq 0$ so as to guarantee $\mathbf{x}+\mathbf{y}$. Since $\mathbb{A}_{n}$ (for $n \geq 3$ ) is not an associative algebra, there always exists an element $z \in \mathbb{A}_{n}$ such that $\left[e_{2}, z, e_{1}\right] \neq 0$. Thus, we obtain $\exists z \in \mathbb{A}_{n},[y, z, x] \neq 0$, where use has been made of $\left[e_{1}, z, e_{1}\right]=0$ by the flexibility of $\mathbb{A}_{n}$.

From Lemma [6] together with Eq. (11), we find that for $x=\left(x_{1}, x_{2}\right)$ in $\mathbb{A}_{n}=\mathbb{A}_{n-1} \times \mathbb{A}_{n-1}$,

$$
x \text { is alternative } \Leftrightarrow x_{1}, x_{2} \text { are alternative with } B_{x_{1}, x_{2}}=0 .
$$

The repeated application of Eqs. (77) and (9) leads to $N_{x}=\|x\|^{2}$ for $x \in \mathbb{A}_{n}$ alternative, so that we obtain

$$
x, y \text { are alternative in } \mathbb{A}_{n} \Rightarrow C_{x, y}^{\prime}=i B_{x, y} .
$$

Definition 7 For $x, y$ in $\mathbb{A}_{n}, V(x, y)$ denotes the vector space generated by the set $\left\{e_{0}, x, y, x y\right\}$, where $e_{0}$ represents the real element of $\mathbb{A}_{n} . V^{\perp}(x, y)$ denotes the orthogonal complement in $\mathbb{A}_{n}=\mathbb{R}^{2^{n}}$.

Lemma 8 For $x, y$ are alternative in $\mathbb{A}_{n}$, we have

1. For $\mathbf{x}+\mathbf{y}, \operatorname{dim}_{\mathbb{R}} V(x, y)=4$;

2. For all $v \in V(x, y), v B_{x, y}=0$;

3. For all $w \in V^{\perp}(x, y), w B_{x, y}=-w \tilde{B}_{x, y}$, where $\tilde{B}_{x, y}:=\left[R_{x}, R_{y}\right]$. 


\section{Proof.}

1. The linear independence of $\left\{e_{0}, x, y, x y\right\}$ is equivalent to that of $\left\{e_{0}, \mathbf{x}, \mathbf{y}, \mathbf{x y}\right\}$, so that we can take $x$ and $y$ to be pure imaginary. Suppose that $c_{0} e_{0}+$ $c_{1} x+c_{2} y+c_{3} x y=0$, where $c_{i} \in \mathbb{R}$. Then from Eq. (2), we obtain $0=$ $c_{0}\left\langle e_{0}, e_{0}\right\rangle=c_{1}\|x\|^{2}+c_{2}\langle x, y\rangle=c_{1}\langle x, y\rangle+c_{2}\|y\|^{2}=c_{0}\langle x, y\rangle+c_{3}\|x\|^{2}\|y\|^{2}$. Thus for $\mathbf{x}+\mathbf{y}$, we get $c_{i}=0(i=0,1,2,3)$, so that the elements in $\left\{e_{0}, x, y, x y\right\}$ are linearly independent over $\mathbb{R}$.

2. It is sufficient to show that $v B_{x, y}=0$ for $v=e_{0}, x, y, x y$. For $v=e_{0}$, this is trivial. For $v=x, y, v B_{x, y}=0$ by definition of $x, y$ being alternative. $(x y) B_{x, y}=-[y, x y, x]=[x, x y, y]=(x(x y)) y-x((x y) y)=\left(x^{2} y\right) y-$ $x\left(x y^{2}\right)=x^{2} y^{2}-x^{2} y^{2}=0$, where in the second equality, we have used the identity $[x, y, z]=-[z, y, x]$ for all $x, y$, and $z$ in $\mathbb{A}_{n}$ [this identity can be derived from the linearization of the flexibility $[x, y, x]=0$ under $x \rightarrow x+\lambda z(\lambda \in \mathbb{R})]$.

3. Since $B_{x, y}$ and $\tilde{B}_{x, y}$ are independent of $\Re(x), \Re(y)$, we can set $x, y$ to be pure imaginary (notice that $w$ is pure imaginary due to $w \perp e_{0}$ ). From $x w+w x=-(x \bar{w}+w \bar{x})=-2\langle x, w\rangle=0, y w+w y=0$, and $x(w y)+(w y) x=x(\overline{y w})+(w y) x=\bar{x}(\overline{w y})+(w y) x=2\langle\bar{x}, w y\rangle=2\langle\bar{x} \bar{y}, w\rangle=$ $2\langle x y, w\rangle=0$, it follows that $w\left(B_{x, y}+\tilde{B}_{x, y}\right)=[x, w, y]+((w x) y-(w y) x)=$ $(x w+w x) y-(x(w y)+(w y) x)=0$.

Notation 9 For $\mathbf{x}$ and $\mathbf{y}$ in $\mathbb{A}_{n} \backslash\left\{e_{0}\right\}, \Delta_{\mathbf{x}, \mathbf{y}}:=\langle\mathbf{x}, \mathbf{y}\rangle I+\frac{1}{2}\left(R_{\mathbf{x}} R_{\mathbf{y}}+R_{\mathbf{y}} R_{\mathbf{x}}\right)$.

In a similar way, as in the proof of Lemma 8 we find that

Lemma 10 For $\mathbf{x}$ and $\mathbf{y}$ are alternative in $\mathbb{A}_{n} \backslash\left\{e_{0}\right\}$,

1. $\Delta_{\mathbf{x}, \mathbf{x}}=\Delta_{\mathbf{y}, \mathbf{y}}=0$;

2. $\forall v \in V(x, y), v \Delta_{\mathbf{x}, \mathbf{y}}=0$.

Notation 11 For $x=\left(x_{1}, x_{2}\right)$ in $\mathbb{A}_{n}=\mathbb{A}_{n-1} \times \mathbb{A}_{n-1}, x_{i_{1} i_{2} \ldots i_{j}}($ for $j=1,2, \ldots)$ is given inductively by $x_{i_{1} \ldots i_{j}}=\left(x_{i_{1} \ldots i_{j} 1}, x_{i_{1} \ldots i_{j} 2}\right)$ in $\mathbb{A}_{n-j}=\mathbb{A}_{n-j-1} \times \mathbb{A}_{n-1-j}$. We denote $x_{k \text { times }}^{11 \ldots 1}$ by $x_{(k)}$.

For $x, y$ are alternative in $\mathbb{A}_{n}$ (with $\mathbf{x} H \mathbf{y}$ ), it is convenient to decompose the vector space $\mathbb{A}_{n}=\mathbb{R}^{2^{n}}$ by using $V(x, y)$ and $V^{\perp}(x, y)$ as

$$
\mathbb{A}_{n}=\bigoplus_{k=1}^{n-1} V_{k}
$$


where

$$
\begin{aligned}
V_{1} & =\left\{r \in \mathbb{A}_{n} \mid r \in V(x, y)\right\}, \\
V_{2} & =\left\{r \in \mathbb{A}_{n} \mid r \in V^{\perp}(x, y) ; r_{1}, r_{2} \in V\left(x_{1}, y_{1}\right)\right\}, \\
V_{3}= & \left\{r \in \mathbb{A}_{n} \mid r \in V^{\perp}(x, y) ; r_{1}, r_{2} \in V^{\perp}\left(x_{1}, y_{1}\right) ; r_{11}, r_{12}, r_{21}, r_{22} \in V\left(x_{11}, y_{11}\right\},\right. \\
& \vdots \\
V_{n-1}= & \bigcap_{k=0}^{n-3}\left\{r \in \mathbb{A}_{n} \mid r_{i_{1} \ldots i_{k}} \in V^{\perp}\left(x_{(k)}, y_{(k)}\right)\right\} \cap\left\{r \in \mathbb{A}_{n} \mid r_{i_{1} \ldots i_{n-2}} \in V\left(x_{(n-2)}, y_{(n-2)}\right)\right\} .
\end{aligned}
$$

Now we evaluate $S_{n}$ for $x$ and $y$ alternative in $\mathbb{A}_{n}$. In this case, $C_{x, y}^{\prime}=i B_{x, y}$ by Eq. (10). Recall that $B_{x, y}$ is anti-symmetric, so that the eigenpolynomial for $i B_{x, y}$ is an even function, and that the eigenvalue of $i B_{x, y}$ is a real number. Thus, $S_{n}$ can be decomposed into two sets: $S_{n}^{+}$and $S_{n}^{-}$, where $S_{n}^{+}\left(S_{n}^{-}\right)$represents the non-negative (non-positive) elements of $S_{n}$ such that $S_{n}=S_{n}^{+} \cup S_{n}^{-}=S_{n}^{+} \cup\left(-S_{n}^{+}\right)$.

Notation 12 For $x, y$ in $\mathbb{A}_{n}$, the set of the eigenvalues of $\left(i B_{x, y}\right)^{2}$ is denoted by $\tilde{S}_{n}$, so that $S_{n}^{+} \cup S_{n}^{+}$is given by square-rooting the elements of $\tilde{S}_{n}$.

From Lemma 8 it is found that $v \in V(x, y)$ is the eigenvector of $\left(i B_{x, y}\right)^{2}$ with its eigenvalue 0 . Thus, the rest we have to do is to obtain the eigenvalue of $\left(i B_{x, y}\right)^{2}$ whose eigenvector belongs to $V^{\perp}(x, y)$. In this case, $\left(i B_{x, y}\right)^{2}$ can be replaced by $\left(i \tilde{B}_{x, y}\right)^{2}$, which can be written using $\Delta_{\mathbf{x}, \mathbf{y}}$ as

$$
\begin{aligned}
\left(i \tilde{B}_{x, y}\right)^{2} & =\left(i \tilde{B}_{\mathbf{x}, \mathbf{y}}\right)^{2} \\
& =2\left(R_{\mathbf{x}} R_{\mathbf{y}} R_{\mathbf{y}} R_{\mathbf{x}}+R_{\mathbf{y}} R_{\mathbf{x}} R_{\mathbf{x}} R_{\mathbf{y}}\right)-\left(R_{\mathbf{x}} R_{\mathbf{y}}+R_{\mathbf{y}} R_{\mathbf{x}}\right)^{2} \\
& =4\left[|\mathbf{x}|^{2}|\mathbf{y}|^{2}-\left(\langle\mathbf{x}, \mathbf{y}\rangle-\Delta_{\mathbf{x}, \mathbf{y}}\right)^{2}\right],
\end{aligned}
$$

where we have used the $\Re(x)$ and $\Re(y)$-independence of $B_{x, y}$ and Lemma [10]i).

The next step is to obtain the recursion formula for $\Delta_{\mathbf{x}, \mathbf{y}}$. The repeated application of Eq. (7) yields for $x \in \mathbb{A}_{n}$

$$
N_{x}=\|x\|^{2} I+\sum_{k=1}^{n-3} \underbrace{\sigma_{0} \otimes \sigma_{0} \otimes \ldots \sigma_{0} \otimes}_{k-1 \text { times }}\left(-i \sigma_{2}\right) \otimes \sum_{i_{1}, \ldots, i_{k-1}} B_{x_{i_{1} \ldots i_{k-1} 1}, x_{i_{1} \ldots i_{k-1} 2}},
$$

where $\sigma_{0}=\left(\begin{array}{ll}1 & 0 \\ 0 & 1\end{array}\right)$ and $i \sigma_{2}=\left(\begin{array}{cc}0 & 1 \\ -1 & 0\end{array}\right)$. The linearization of $N_{\mathbf{x}, \mathbf{x}}(=$ $\left.R_{\mathbf{x}, \overline{\mathbf{x}}}=-R_{\mathbf{x}, \mathbf{x}}\right)$ by $\mathbf{x} \rightarrow \mathbf{x}+\lambda \mathbf{y} \in \mathbb{A}_{n} \backslash\left\{e_{0}\right\}$ leads to

$$
2 \Delta_{\mathbf{x}, \mathbf{y}}=\sum_{k=1}^{n-3} \underbrace{\sigma_{0} \otimes \sigma_{0} \otimes \ldots \sigma_{0} \otimes}_{k-1 \text { times }}\left(-i \sigma_{2}\right) \otimes \sum_{i_{1}, \ldots, i_{k-1}}\left(B_{\mathbf{x}_{i_{1} \ldots i_{k-1}}, \mathbf{y}_{i_{1} \ldots i} \ldots-1^{2}}+(\mathbf{x} \leftrightarrow \mathbf{y})\right) .
$$


Recall that we are dealing with the case of $x, y$ being alternative in $\mathbb{A}_{n}$. Then from Lemma[ and Eq. (9), we find that $\mathbf{x}_{i_{1} \ldots i_{k-1} 1} \| \mathbf{x}_{i_{1} \ldots i_{k-1} 2}$, and the analogous relation for $\mathbf{y}$, so that we can set

$$
\mathbf{x}_{\underbrace{11 \ldots 1}_{k-1} 2}=\kappa_{1}^{(k)} \mathbf{x}_{(k)}, \quad \mathbf{y}_{\underbrace{11 \ldots 1}_{k-1} 2}=\kappa_{2}^{(k)} \mathbf{y}_{(k)},
$$

where $\kappa_{1}^{(k)}, \kappa_{2}^{(k)} \in \mathbb{R}($ for $k=1,2, \ldots, n-3)$ are certain parameters. Substituting Eq. (14) into Eq. (13), we obtain

$$
2 \Delta_{\mathbf{x}, \mathbf{y}}=\sum_{k=1}^{n-3} a_{(k)} \prod_{i=1}^{k-1} b_{(i)} \underbrace{\sigma_{0} \otimes \ldots \sigma_{0} \otimes}_{k-1 \text { times }}\left(i \sigma_{2}\right) \otimes B_{\mathbf{x}_{(k)}, \mathbf{y}_{(k)}},
$$

where $\prod_{i=1}^{0} b_{(i)}$ stands for 1 , and $a_{(k)}, b_{(i)}$ are given by

$$
a_{(k)}=\kappa_{2}^{(k)}-\kappa_{1}^{(k)}, \quad b_{(i)}=1+\kappa_{1}^{(i)} \kappa_{2}^{(i)} .
$$

From Eq. (15), the recursion formula for $\Delta_{\mathbf{x}, \mathbf{y}}$ can be written as

$$
2 \Delta_{\mathbf{x}_{(k-1)}, \mathbf{y}_{(k-1)}}=a_{(k)} i \sigma_{2} \otimes B_{\mathbf{x}_{(k)}, \mathbf{y}_{(k)}}+2 b_{(k)} \sigma_{0} \otimes \Delta_{\mathbf{x}_{(k)}, \mathbf{y}_{(k)}} \quad(k=1,2, \ldots, n-3),
$$

where $\mathbf{x}_{(0)}$ denotes $\mathbf{x}$ in $\mathbb{A}_{n} \backslash\left\{e_{0}\right\}$. Note that Eq. (16) is analogous to Eq. (77). Recall the determinant identity $\left|\sigma_{0} \otimes A+i \sigma_{2} \otimes B\right|=|A+i B||A-i B|$. Then, we find that all the eigenvalues of $\Delta_{\mathbf{x}_{(k-1)}, \mathbf{y}_{(k-1)}}$ remain invariant, even if $\Delta_{\mathbf{x}_{(k-1)}, \mathbf{y}_{(k-1)}}$ is replaced by

$$
\Delta_{\mathbf{x}_{(k-1)}, \mathbf{y}_{(k-1)}} \rightarrow \tilde{\Delta}_{\mathbf{x}_{(k)}, \mathbf{y}_{(k)}}:= \pm \frac{1}{2} a_{(k)} i B_{\mathbf{x}_{(k)}, \mathbf{y}_{(k)}}+b_{(k)} \Delta_{\mathbf{x}_{(k)}, \mathbf{y}_{(k)}} .
$$

From Lemmas 8 and 10] it follows that

$r \tilde{\Delta}_{\mathbf{x}_{(k)}, \mathbf{y}_{(k)}}= \begin{cases}0 & {\left[\text { for } r \in V\left(x_{(k)}, y_{(k)}\right)\right],} \\ \pm \frac{1}{2} a_{(k)} \sqrt{\left(i \tilde{B}_{\mathbf{x}_{(k)}, \mathbf{y}_{(k)}}\right)^{2}}+b_{(k)} \Delta_{\mathbf{x}_{(k)}, \mathbf{y}_{(k)}} & {\left[\text { for } r \in V^{\perp}\left(x_{(k)}, y_{(k)}\right)\right],}\end{cases}$

where $\left(i \tilde{B}_{\mathbf{x}_{(k)}, \mathbf{y}_{(k)}}\right)^{2}$ can be rewritten using $\Delta_{\mathbf{x}_{(k)}, \mathbf{y}_{(k)}}$ as in Eq. (12). Eventually, all the eigenvalues of $\Delta_{\mathbf{x}_{(k-1)}, \mathbf{y}_{(k-1)}}$ are given inductively by those of $\Delta_{\mathbf{x}_{(k)}, \mathbf{y}_{(k)}}$.

Proposition 13 For $x, y$ alternative in $\mathbb{A}_{n}$, the sets $S_{n}($ for $n=0,1, \ldots)$ satisfy the inclusion relation

$$
S_{0} \subset S_{1} \subset S_{2} \subset \ldots
$$


Proof. It is sufficient to show that $\tilde{S}_{n-1} \subset \tilde{S}_{n}$ for $n=1,2, \ldots$, because $S_{n}$ is given by square-rooting the element of $\tilde{S}_{n}$. In the case of $\mathbf{x} \| \mathbf{y}$ for $x, y$ in $\mathbb{A}_{n}$, where $B_{\mathbf{x}, \mathbf{y}}=0$ by Lemma [6] all the elements of $\tilde{S}_{n}$ (hence those of $S_{n}$ ) turn out to be vanishing, so that the Proposition is trivial in this case. In what follows, we assume that $\mathbf{x}+\mathbf{y}$.

We decompose the vector space $\mathbb{A}_{n}$ as in Eq. (11). For all $r \in V_{1}=V(x, y)$, where $\operatorname{dim} V_{1}=4$, we obtain $r B_{x, y}=0$ by Lemma 8 ii), so that $V_{1}$ represents the eigenspace of $\left(i B_{x, y}\right)^{2}$ whose eigenvalue is zero. It should be recalled here that $B_{x, y}=0$ for all $x, y$ in $\mathbb{A}_{2}$ due to the associativity of $\mathbb{A}_{2}$, so that $\tilde{S}_{2}=\{0,0,0,0\}$. Hence, the eigenvalue of $\left(i B_{x, y}\right)^{2}$ whose eigenvector belongs to $V_{1}$ is given by $\tilde{S}_{2}$.

If the eigenvector $r=\left(r_{1}, r_{2}\right)$ in $\mathbb{A}_{n}=\mathbb{A}_{n-1} \times \mathbb{A}_{n-1}$ of $\left(i B_{x, y}\right)^{2}$ belongs to $V^{\perp}(x, y)$, its eigenvalue turns out to be the eigenvalue of $\left(i \tilde{B}_{x, y}\right)^{2}$ by Lemma 8 iii), so that we have only to calculate the eigenvalue of $\Delta_{\mathbf{x}, \mathbf{y}}$, hence that of $\tilde{\Delta}_{\mathbf{x}_{1}, \mathbf{y}_{1}}$. If $r$ further belongs to $V_{2}$ (where $\operatorname{dim} V_{2}=4$ ) so that $r_{1}, r_{2} \in V\left(x_{1}, y_{1}\right)$, the eigenvalue of $\tilde{\Delta}_{\mathbf{x}_{1}, \mathbf{y}_{1}}$ turns out to be vanishing by Eq. (17) with $k=1$. On the other hand, the relation of $\Delta_{\mathbf{x}, \mathbf{y}}=0$, in itself, holds for all $\mathbf{x}, \mathbf{y}$ in $\mathbb{A}_{3}$ (where $\operatorname{dim} \mathbb{A}_{3}=8$ ) by Eq. (15) or (16). Thus, the four eigenvalues of $\left(i B_{x, y}\right)^{2}$ whose corresponding eigenvectors belong to $V_{2}$ are given by the four elements in $\tilde{S}_{3}$. Recall that among the elements of $\tilde{S}_{3}$, the eigenvalue whose eigenvector belongs to $V_{1}$ is given by $\tilde{S}_{2}$, so that the eigenvalue of $\left(i B_{x, y}\right)^{2}$ whose eigenvalue belongs to $V_{2}$ is given by $\tilde{S}_{3} \backslash \tilde{S}_{2}$.

In a similar way, we repeatedly apply Eqs. (16) and (17) and use the identity $\Delta_{\mathbf{x}_{(n-3)}, \mathbf{y}_{(n-3)}}=0$, to find that the eigenvalue of $\left(i B_{x, y}\right)^{2}$ whose eigenvector belongs to $V_{k}(k=2,3, \ldots, n-1)$ is given by $\tilde{S}_{k+1} \backslash \tilde{S}_{k}$, as is summarized in Table 2. Thus, we obtain the inclusion relation $\tilde{S}_{k} \subset \tilde{S}_{k+1}$ for $k=2,3, \ldots, n-1$. Since $n$ can be chosen as any $n \geq 3$, we get $\tilde{S}_{k} \subset \tilde{S}_{k+1}$ for $k \geq 2$ (for $k=0,1$, the above inclusion relation is found to be satisfied from the direct calculation of $S_{k}$ ).

Table 2: Eigenvalues of $\left(i B_{x, y}\right)^{2}$ for $x, y$ alternative in $\mathbb{A}_{n}=\bigoplus_{k=1}^{n-1} V_{k}$, with $\mathbf{x}+\mathbf{y}$.

\begin{tabular}{ccc}
\hline Eigenspace & Eigenvalues & $\operatorname{dim} V_{i}$ \\
\hline$V_{1}$ & $\tilde{S}_{2}$ & 4 \\
$V_{2}$ & $\tilde{S}_{3} \backslash \tilde{S}_{2}$ & 4 \\
$V_{3}$ & $\tilde{S}_{4} \backslash \tilde{S}_{3}$ & 8 \\
$\vdots$ & $\vdots$ & $\vdots$ \\
$V_{n-1}$ & $\tilde{S}_{n} \backslash \tilde{S}_{n-1}$ & $2^{n-1}$ \\
\hline
\end{tabular}


In the final analysis, we have found from Eqs. (8) and (10) that for $x, y$ alternative in $\mathbb{A}_{n}$, the eigenequation for $N_{(x, y)}$ is replaced by

$$
r N_{(x, y)}=\left(\|x\|^{2}+\|y\|^{2}+\beta\right) r \Longleftrightarrow d\left(i B_{x, y}\right)=\beta d,
$$

where $\beta \in S_{n}$ and $d=r_{1}+i r_{2}$ or $d=r_{2}-i r_{1}$ with $r=\left(r_{1}, r_{2}\right)$ in $\mathbb{A}_{n+1}=\mathbb{A}_{n} \times \mathbb{A}_{n}$. Noticing that $d\left(i B_{x, y}\right)^{2}=\beta^{2} d$ so that $r_{i}\left(i B_{x, y}\right)^{2}=\beta^{2} r_{i}$ (for $\left.i=1,2\right)$, and that $\beta^{2} \in \tilde{S}_{n} \Leftrightarrow \beta \in S_{n}$, we find from Table 2 that

$$
\begin{aligned}
& r_{1}, r_{2} \in V_{1} \Longrightarrow \beta \in S_{2}, \\
& r_{1}, r_{2} \in V_{k} \Longrightarrow \beta \in S_{k+1} \backslash S_{k} \quad(\text { for } k=2,3, \ldots, n-1) .
\end{aligned}
$$

\section{$3 \quad$ Reciprocity}

In this section, we show how the invariant under the reciprocal transformation simplifies the functional form of $\beta \in S_{n}$. Recall that $\beta$ is a parameter such that represents the violation of the rotational symmetry breaking of $R_{(x, y)}$. Actually, we have $\left\|r R_{(x, y)}\right\|^{2}=(1+\beta)\|r\|^{2}$ for $\|x\|^{2}+\|y\|^{2}=1$, so that $\beta=0$ corresponds to the rotational symmetry of $R_{(x, y)}$.

Before proceeding further, we consider the case of maximal violation of the rotational symmetry of $R_{(x, y)}$. This consideration will give us some hints of how to apply the reciprocity. The maximal violation is realized for $\left\|r R_{(x, y)}\right\|=0$, that is, for $\|x\|^{2}+\|y\|^{2}+\beta=0$. This occurs for $\beta \in S_{n} \backslash S_{2}$, with $\beta$ written from Eq. (12) as $-2 \sqrt{\|\mathbf{x}\|^{2}\|\mathbf{y}\|^{2}-(\langle\mathbf{x}, \mathbf{y}\rangle-\delta)^{2}}$, where $\delta$ represents the eigenvalues of $\Delta_{\mathbf{x}, \mathbf{y}}$ (the case for $\beta=+2 \sqrt{\cdots}$ is not appropriate). Thus from $\left(\|x\|^{2}+\|y\|^{2}\right)^{2}=$ $\beta^{2}, x$ and $y$ should satisfy

$$
\Re(x)=\Re(y)=\|\mathbf{x}\|-\|\mathbf{y}\|=\langle\mathbf{x}, \mathbf{y}\rangle-\delta=0 .
$$

Here, we have a practical question of which of the eigenvalues $\delta$ we should choose. To restrict the choice of $\delta$, it is convenient to use the invariance under the reciprocal transformation. Notice that Eq. (18) is derived from the invariance of

$$
(\Re(x), \Re(y)),\|\mathbf{x}\|-\|\mathbf{y}\|,\langle\mathbf{x}, \mathbf{y}\rangle-\delta
$$

under the reciprocal transformation $(x, y) \mapsto(-y, x)$, where $\delta$ is transformed to $(-\delta)$ due to $\Delta_{-\mathbf{y}, \mathbf{x}}=-\Delta_{\mathbf{x}, \mathbf{y}}$. Furthermore, the invariance of $\|\mathbf{x}\|-\|\mathbf{y}\|$ under $(x, y) \mapsto(-y, x)$ implies the invariance of each term, that is, $\|\mathbf{x}\|$ and $\|\mathbf{y}\|$. However, the invariance of $\langle\mathbf{x}, \mathbf{y}\rangle-\delta$ does not imply the invariance of each term, that is, $\langle\mathbf{x}, \mathbf{y}\rangle$ and $\delta$, unless $\langle\mathbf{x}, \mathbf{y}\rangle=\delta=0$. Hence, the invariance of $\delta$ leads to $\delta=0$. This is our choice of $\delta$ for the case of maximal violation, where $\langle\mathbf{x}, \mathbf{y}\rangle=0$ is required.

Even for a general case, we will require $\langle\mathbf{x}, \mathbf{y}\rangle=0$, which is obtained from the invariance of $\langle\mathbf{x}, \mathbf{y}\rangle$ under $(x, y) \mapsto(-y, x)$. In a similar way, the invariance of $\left\langle\mathbf{x}_{1}, \mathbf{y}_{1}\right\rangle$ under $(x, y) \mapsto(-y, x)$ implies $\left\langle\mathbf{x}_{1}, \mathbf{y}_{1}\right\rangle=0$. In this case, $\Re\left(x_{2}\right) \Re\left(y_{2}\right)=$ 
0 is required from the decomposition $\langle\mathbf{x}, \mathbf{y}\rangle=\left(1+b_{(1)}\right)\left\langle\mathbf{x}_{1}, \mathbf{y}_{1}\right\rangle+\Re\left(x_{2}\right) \Re\left(y_{2}\right)$. Furthermore, the invariance of $\Re\left(x_{2}\right) \Re\left(y_{2}\right)=0$, namely, $\Re\left(x_{2}\right)=0$ or $\Re\left(y_{2}\right)=0$ under $(x, y) \mapsto(-y, x)$ implies that $\Re\left(x_{2}\right)=\Re\left(y_{2}\right)=0$. In this case, we have $|\mathbf{x}|^{2}|\mathbf{y}|^{2}=\left(a_{(1)}^{2}+b_{(1)}^{2}\right)\left|\mathbf{x}_{1}\right|^{2}\left|\mathbf{y}_{1}\right|^{2}$. In an analogous way, we obtain from the invariance of the reciprocal transformation, $\left\langle\mathbf{x}_{11}, \mathbf{y}_{11}\right\rangle=\Re\left(x_{12}\right)=\Re\left(y_{12}\right)=0$ and so forth, so that

$$
\begin{cases}\left\langle\mathbf{x}_{(k)}, \mathbf{y}_{(k)}\right\rangle=0 & (\text { for } k=0,1, \ldots, n-3), \\ \left|\mathbf{x}_{(k-1)}\right|^{2}\left|\mathbf{y}_{(k-1)}\right|^{2}=\left(a_{(k)}^{2}+b_{(k)}^{2}\right)\left|\mathbf{x}_{(k)}\right|^{2}\left|\mathbf{y}_{(k)}\right|^{2} & (\text { for } k=1,2, \ldots, n-3) .\end{cases}
$$

Under the condition Eq. (20), it is convenient to define $\sin \theta_{k}$ and $\cos \theta_{k}$ by

$$
\sin \theta_{k}=\frac{a_{(k)}}{\sqrt{a_{(k)}^{2}+b_{(k)}^{2}}}, \quad \cos \theta_{k}=\frac{b_{(k)}}{\sqrt{a_{(k)}^{2}+b_{(k)}^{2}}} .
$$

Substituting Eq. (20) into Eq. (17) and using Eqs. (12) and (21), we eventually obtain $\tilde{S}_{n}$ (whose elements are quadruply degenerated) in a simple form as

$$
\begin{aligned}
\tilde{S}_{3}^{\prime} \backslash \tilde{S}_{2}^{\prime} & =\left\{\Delta^{2}\right\}, \\
\tilde{S}_{4}^{\prime} \backslash \tilde{S}_{3}^{\prime} & =\left\{\Delta_{+}^{2}, \Delta_{-}^{2}\right\}, \\
\tilde{S}_{5}^{\prime} \backslash \tilde{S}_{4}^{\prime} & =\left\{\Delta_{++}^{2}, \Delta_{+-}^{2}, \Delta_{-+}^{2}, \Delta_{--}^{2}\right\}, \\
\ldots &
\end{aligned}
$$

where $\tilde{S}_{n}=\bigcup_{i=1}^{4} \tilde{S}_{n}^{\prime}$ and $\Delta_{\underbrace{+\ldots \pm}_{\text {times }}}=\Delta \cdot \cos \left( \pm \theta_{1} \pm \theta_{2} \pm \ldots \pm \theta_{k}\right)$, with $\Delta=2 \mid \mathbf{x} \times$ $\mathbf{y} \mid$. Note that $(x, y) \mapsto(-y, x) \Rightarrow\left(\kappa_{1}^{(k)}, \kappa_{2}^{(k)}\right) \mapsto\left(\kappa_{2}^{(k)}, \kappa_{1}^{(k)}\right) \Rightarrow\left(a_{(k)}, b_{(k)}\right) \mapsto$ $\left(-a_{(k)}, b_{(k)}\right) \Rightarrow \theta_{k} \mapsto-\theta_{k}$ for $k=1,2, \ldots, n-3$, so that $\Delta_{ \pm \ldots \pm} \mapsto \Delta_{\mp \ldots \mp}(=$ $\left.\Delta_{ \pm \ldots \pm}\right)$, as is expected from the invariance of $\left(i B_{x, y}\right)^{2}$ under $(x, y) \mapsto(-y, x)$.

\section{Application}

Now we examine whether or not the simplified $\beta$ is available as an order parameter in some isometry (rotational symmetry) breaking system. Consider the electro-weak gauge boson system, where the $U(1) \times S U(2)$ gauge symmetry is (partially) broken. The reason of adopting this system is that the gauge fields have a hierarchical structure $\mathbf{1} \oplus \mathbf{1} \oplus \mathbf{2}$, which is analogous to $V_{1} \oplus V_{2} \oplus V_{3}$, as

$$
\begin{aligned}
\mathbb{R}^{16} & =\mathbb{R}^{4} \oplus \mathbb{R}^{4} \oplus \mathbb{R}^{8} \\
& =A_{\mu} \oplus Z_{\mu} \oplus W_{\mu}^{ \pm},
\end{aligned}
$$

where $A_{\mu}, Z_{\mu}$, and $W_{\mu}^{ \pm}$represent the photon, Z-boson, and W-boson fields, respectively (we treat them as classical, not quantized). At low energy, when the gauge symmetry is broken, the gauge boson turns out to be massive. Denote by $\phi$ the mass matrix of the gauge boson, that is, $\phi: \mathbb{R}^{16} \rightarrow \mathbb{R}^{16}$ by 
$\left(A_{\mu}, Z_{\mu}, W_{\mu}^{+}, W_{\mu}^{-}\right) \phi=\left(0, m_{\mathrm{z}} Z_{\mu}, m_{\mathrm{w}} W_{\mu}^{+}, m_{\mathrm{w}} W_{\mu}^{-}\right)$, where $m_{\mathrm{z}}$ and $m_{\mathrm{w}} \pm$ represent the $\mathrm{Z}$ and $\mathrm{W}$-boson masses, respectively. In this case, the map from the gauge field to the corresponding mass is injective. On the other hand, recall that $\mathbb{R}^{16}$ is decomposed into

$$
\mathbb{R}^{16}=V_{1} \oplus V_{2} \oplus V_{3},
$$

where the map from $V_{i}$ to the eigenvalue of $i B_{x, y}$ is injective, as long as $0, \Delta$, and $\Delta_{+}$are not degenerate in magnitude. Comparing Eqs. (22) and (23), we may identify the gauge field with $V_{i}$, so that we have the following one-to-one correspondence:

$$
\begin{array}{ccc}
\left(A_{\mu}, Z_{\mu}, W_{\mu}^{ \pm}\right) & \longleftrightarrow & \left(V_{1}, V_{2}, V_{3}\right) \\
\phi \downarrow & \downarrow \\
\left(0, m_{\mathrm{z}} Z_{\mu}, m_{\mathrm{w}} W_{\mu}^{ \pm}\right) & & \left(0, \Delta \cdot B_{x, y}, \Delta_{ \pm} \cdot V_{3}\right) .
\end{array}
$$

As a consequence, there exists a one-to-one map $\pi: \mathbb{R} \rightarrow \mathbb{R}$ such that

$$
\left(0, m_{\mathrm{z}}, m_{\mathrm{w}}\right) \stackrel{\pi}{\longrightarrow}\left(0, \Delta, \Delta_{+}\right),
$$

where it should be noted that $\Delta_{+}=\Delta_{-}$.

The rest we will show is that we can set $\pi(x)=x^{a}(a>0)$ under some reasonable assumption. From the boundary condition $\pi(0)=0$ and the monomorphism of $\pi(x)$, there exist $x_{0}, c_{0} \in \mathbb{R} \backslash\{0\}$ such that $\pi\left(x_{0}\right)=c_{0}$, so that (after the scaling of $\left.x \rightarrow x / x_{0}, \pi(x) \rightarrow \pi(x) / c_{0}\right)$ there is no loss of generality that we take $\pi(1)=1$. Suppose that if $m_{\mathrm{z}}$ scales as $m_{\mathrm{z}} \rightarrow \lambda m_{\mathrm{z}}(\lambda \in \mathbb{R})$, then $m_{\mathrm{w}}$ similarly scales as $m_{\mathrm{w}} \rightarrow \lambda m_{\mathrm{w}}$. This condition can be interpreted, based on the standard model, as follows. While the gauge-boson mass is proportional to the vacuum expectation value $v$ of the Higgs field, the ratio $m_{\mathrm{w}} / m_{\mathrm{z}}$ is independent of $v$ it depends on the coupling constants $\left(g, g^{\prime}\right)$ between the Higgs and gauge bosons]. If $v$ and $\left(g, g^{\prime}\right)$ can be regarded as independent parameters, $m_{\mathrm{w}} / m_{\mathrm{z}}$ remains invariant under the scaling $v \rightarrow \lambda v$. This is the physical meaning of the scaling $m_{\mathrm{z}} \rightarrow \lambda m_{\mathrm{z}}$. Substituting $m_{\mathrm{z}} \rightarrow \lambda m_{\mathrm{z}}$ and $m_{\mathrm{w}} \rightarrow \lambda m_{\mathrm{w}}\left(\right.$ with $\left.\lambda=m_{\mathrm{z}}^{-1}\right)$ into the relation $\pi\left(m_{\mathrm{w}}\right)=\Delta_{+}=\pi\left(m_{\mathrm{z}}\right) \cos \theta_{1}$ to eliminate $\cos \theta_{1}$, we obtain $\pi\left(m_{\mathrm{w}}\right)=\pi\left(m_{\mathrm{z}}\right) \pi\left(m_{\mathrm{w}} / m_{\mathrm{z}}\right)$, where use has been made of $\pi(1)=1$. Suppose further that this relation holds for all $m_{\mathrm{w}}, m_{\mathrm{z}}$ in $\mathbb{R}$. Then, we find from the boundary conditions $\pi(0)=0$ and $\pi(1)=1$, that

$$
\pi(x)=x^{a} \quad(\text { with } a>0),
$$

where $a$ is a constant which amounts to $\mathrm{d} \pi(x) /\left.\mathrm{d} x\right|_{x=1}$.

The prediction of Eq. (24) is that, as in the standard model, $m_{\mathrm{w}}$ is less than $m_{\mathrm{z}}$ :

$$
\cos \theta_{1}=\frac{\pi\left(m_{\mathrm{w}}\right)}{\pi\left(m_{\mathrm{z}}\right)}=\left(\frac{m_{\mathrm{w}}}{m_{\mathrm{z}}}\right)^{a} .
$$

It is apparent that $\theta_{1}=\theta_{\mathrm{w}}$ for $a=1$, where $\theta_{\mathrm{w}}$ represents the Weinberg angle, although the value of $a$ in itself is not specified at the present stage. 


\section{Summary}

We have calculated the eigenvalue of the right multiplication $R_{(x, y)}$ for $x$ and $y$ alternative in the Cayley-Dickson algebra $\mathbb{A}_{n}$, by calculating, instead, the eigenvalue $\beta$ of $i B_{x, y}$. The eigenvalue $\beta$ represents the violation of the rotational symmetry of $R_{(x, y)}$. The requirement of the alternative entries guarantees that half of the eigenvectors of $N_{x}$ in $\mathbb{A}_{n}$ are still eigenvectors in the subspace which is isomorphic to $\mathbb{A}_{n-1}$. In calculating $\beta$, the essential point lies in Lemma 8 iii). Here, we comment on this property from a Lie algebraic point of view. Denote $\left[L_{x}, L_{y}\right]$ by $\tilde{B}_{x, y}^{\prime}$. Then, it is analogous to show that $w B_{x, y}=-w \tilde{B}_{x, y}^{\prime}$ for $w \in$ $V^{\perp}(x, y)$, as in Lemma 8 8 iii), so that we obtain $w B_{x, y}=-w D_{x, y}$, where $D_{x, y}=$ $B_{x, y}+\tilde{B}_{x, y}+\tilde{B}_{x, y}^{\prime}$. It should be noted that $D_{x, y}$ is a derivation of an alternative algebra. The derivation $D$ of an algebra $\mathfrak{A}$ is defined by $(x y) D=(x D) y+x(y D)$ for all $x, y$ in $\mathfrak{A}$. If $\mathfrak{A}$ is an alternative algebra, $D$ is given by the sum $\sum D_{x_{i}, y_{i}}$ for $x_{i}, y_{i}$ in $\mathfrak{A}$ [1. Thus, $D$ forms a Lie algebra, so that the corresponding Lie group turns out to be isometric. Hence, we obtain $\|w\|=\left\|w e^{\theta D_{x, y}}\right\|=\left\|w e^{-\theta B_{x, y}}\right\|$ for $w \in V^{\perp}(x, y)$ and $\theta \in \mathbb{R}$. Recall that $v=v e^{-\theta B_{x, y}}$ for $v \in V(x, y)$ by Lemma $8 \mathrm{ii}$ ). Then, we find that the map $e^{-\theta B_{x, y}}: \mathbb{A}_{n} \rightarrow \mathbb{A}_{n}$ is isometric in $\mathbb{A}_{n}$. The subspace of $\mathbb{A}_{n+1}$ with (without) the rotational symmetry of $R_{(x, y)}$ in $\mathbb{A}_{n+1}$ is invariant (transformed) under the isometric transformation $e^{-\theta B_{x, y}}$ in $\mathbb{A}_{n}$.

The reciprocity is useful in simplifying the functional form of $\beta$. Assume that each term in Eq. (19) is invariant under the reciprocal transformation $(x, y) \mapsto$ $(-y, x)$. Then, it follows that $\langle\mathbf{x}, \mathbf{y}\rangle=0$. In this case, together with some other invariants, $\beta$ is simply written as $\Delta_{ \pm \ldots \pm}$. This simplified expression would reduce further analysis. In connection to (infinite dimensional) Lie algebra, it may be interesting to point out that one of the solutions (called $R$ matrix) to the Yang-Baxter equation is written using $\Delta_{ \pm \ldots \pm}$ as

$$
R=\frac{1}{\Delta}\left(\begin{array}{cccc}
\Delta_{+} & 0 & 0 & 0 \\
0 & \Delta_{++} & \Delta_{+++} & 0 \\
0 & \Delta_{+++} & \Delta_{++} & 0 \\
0 & 0 & 0 & \Delta_{+}
\end{array}\right)
$$

with $\theta_{1}+2 \theta_{2}+\theta_{3}=0$.

If some physical field $S$ with an isometry breaking has a hierarchical structure $\mathbf{1} \oplus \mathbf{1} \oplus \mathbf{2} \oplus \mathbf{4} \oplus \ldots$, with an injective map from the field to a scalar quantity $m$ (called an "order parameter"), then there is a one-to-one map $\pi: m \mapsto \beta$. One example is realized in the electro-weak gauge boson field, where $\mathbf{1} \oplus \mathbf{1} \oplus \mathbf{2}=$ $A_{\mu} \oplus Z_{\mu} \oplus W_{\mu}^{ \pm}$, with an injective map from the gauge field to the corresponding mass. In this case, the one-to-one map $\pi$ implies that (under some reasonable condition) the $\mathrm{W}$-boson mass is less than the Z-boson mass, as in the standard model.

Another example may be found in the light meson field, where the $S U(3)$ flavor symmetry is breaking. The octet $\mathbf{8}$ in the light mesons $\mathbf{3} \otimes \overline{\mathbf{3}}(=\mathbf{8} \oplus \mathbf{1})$, 
forming a 32-dimensional vector space, has a hierarchical structure

$$
\begin{aligned}
\mathbf{8} & =\mathbf{1} \oplus \mathbf{1} \oplus \mathbf{2} \oplus \mathbf{4} \\
& =\eta \oplus \pi^{0} \oplus\left(\pi^{+}, \pi^{-}\right) \oplus\left(K^{+}, K^{-}, K^{0}, \bar{K}^{0}\right),
\end{aligned}
$$

where $\eta, \pi$, and $K$ represent the corresponding meson fields. Recalling that $\mathbb{R}^{32}$ can be decomposed into $V_{1} \oplus V_{2} \oplus V_{3} \oplus V_{4}$, we find that there is a one-to-one correspondence between $\beta$ and the meson mass. The detailed analysis of the meson mass is beyond the scope of the present paper, which, however, will be discussed elsewhere.

\section{Acknowledgments}

The authors are indebted to K. Terada and M. Terauchi for their stimulating discussion.

\section{References}

\section{References}

[1] R. D. Schafer, Introduction to Non-Associative Algebras (Dover, New York, 1995).

[2] M. Born, Proc. Roy. Soc. London A 165 (1938) 291; Rev. Mod. Phys. 21 (1949) 463.

[3] M. Günaydin and F. Gürsey, J. Math. Phys. 14 (1973) 1651.

[4] J. Soucek, J. Phys. A 14 (1981) 1629.

[5] T. Kugo and P. Townsend, Nucl. Phys. B 221 (1983) 357.

[6] J. M. Evans, Nucl. Phys. B 298 (1988) 92.

[7] G. M. Dixon, Division Algebras: Octonions, Quaternions, Complex Numbers and the Algebraic Design of Physics (Kluwer, Dordrecht, 1994).

[8] J. Lõhmus, E. Paal, and L. Sorgsepp, Nonassociative Algebras in Physics (Hadronic Press, Palm Harbor, Florida, 1994).

[9] S. L. Adler, Quaternionic Quantum Mechanics and Quantum Fields (Oxford Univ. Press, Oxford, 1995).

[10] S. Okubo, Introduction to Octonions and Other Non-Associative Algebras in Physics (Cambridge Univ. Press, Cambridge, 1995).

[11] F. Gürsey and C.-H. Tze, On the Role of Division, Jordan, and Related Algebras in Particle Physics (World Scientific, Singapore, 1996). 
[12] L. Sorgsepp and J. Lõhmus, Hadronic J. 4 (1981) 327.

[13] J. Baez, Bull. Amer. Math. Soc. 39 (2002) 145; math.RA/0105155.

[14] G. Moreno, Bol. Soc. Mat. Mexicana (3) 4 (1998) 13; q-alg/9710013

[15] R. D. Schafer, Am. J. Math. 76 (1954) 435.

[16] S. Kuwata, Int. J. Mod. Phys. A 19 (2004) 1525; hep-th/0306271 Note that $B(x, y)$ in [16] corresponds to $\left(-B_{x, y}\right)$ in the present paper. 